\title{
Theoretical Treatment of Cholesterol Monohydrate Dissolution in Renex- 690/Ionic Surfactant Systems Based on a Physical Model Approach ${ }^{1}$
}

\author{
NORMAN F. H. HO, ${ }^{* 2}$ SHANKER L. GUPTA, $\dagger$ AND WILLIAM I. HIGUCHI \\ *College of Pharmacy, the University of Michigan, Ann Arbor, Michigan 48109; †E. R. Squibb, New Brunswick, \\ New Jersey 08903; and $\ddagger$ Department of Pharmaceutics, College of Pharmacy, \\ University of Utah, Salt Lake City, Utah 84112
}

Received August 17, 1983; accepted April 13, 1984

\begin{abstract}
The dissolution kinetics of cholesterol monohydrate in the presence of Renex-690 and ionic surface active agents (benzalkonium chloride and sodium dodecyl sulfate) are dramatically influenced by the addition of $\mathrm{NaCl}$. The mass transfer resistances decreased with increasing electrolyte concentrations and attained an asymptotic value predicted for the aqueous boundary layer-controlled situation. Electrophoretic mobility studies showed that the mixed surfactants are adsorbed on the originally negatively charged cholesterol surface to an extent that the effective surface charge is attributed to the adsorbed surface active molecules. The results suggest that electrical repulsion between charged micelles and the cholesterol surface plays a dominant role in the mechanistic interpretation of the interfacial barrier to dissolution. A model embodying the classical Smoluchowski flocculation theory of colloidal particles and the DLVO potential energy theory of electrical double layer repulsion and dispersion attraction was applied to the dissolution data to obtain a quantitative description of the interfacial barrier in terms of surface charge densities, electrical double layer parameters, surface potentials, and dispersion force constants.
\end{abstract}

\section{INTRODUCTION}

Our previous studies on the dissolution kinetics of cholesterol monohydrate pellets in the presence of Renex-690 (polyoxyethylene[10]nonylphenol ether) and ionic surface active agents (e.g., benzalkonium chloride and sodium dodecyl sulfate) have shown that the kinetics are controlled by an interfacial barrier and are dramatically influenced by the addition of sodium chloride (1). Figures 1 and 2 show the change in the total mass transfer resistance with electrolyte concentration for cholesterol monohydrate in micellar solutions composed of $1 \%$ Renex-690/6.8 $\mathrm{m} M$ benzalkonium chloride and $1 \%$ Renex$690 / 6.8 \mathrm{~m} M \mathrm{Na}$ dodecyl sulfate and multiple concentrations thereof. It is seen that the mass transfer resistance $R$ decreases with

\footnotetext{
${ }^{1}$ Work supported by NIAMDD Grants AMI6694 and AM32472.

${ }^{2}$ To whom correspondence should be addressed.
}

increasing electrolyte concentrations and attains an asymptotic value predicted for the aqueous boundary layer-controlled situation. Furthermore, the electrophoretic mobility studies in Fig. 3 show that Renex-690 and added surface active ions are adsorbed on the originally negatively charged cholesterol surface to an extent that the effective surface charge is attributed to the adsorbed surface active agents. The overall results suggest that electrical repulsion between charged micelles and the cholesterol surface plays an important, if not dominant, role in the mechanistic interpretation of the interfacial barrier of the dissolution process.

In this presentation we describe a physical model embodying the classical Smoluchowski flocculation theory of colloidal particles and the Verwey and Overbeek potential energy theory of electrical double layer repulsion and dispersion attraction. Subsequently, the model will be applied to the dissolution 


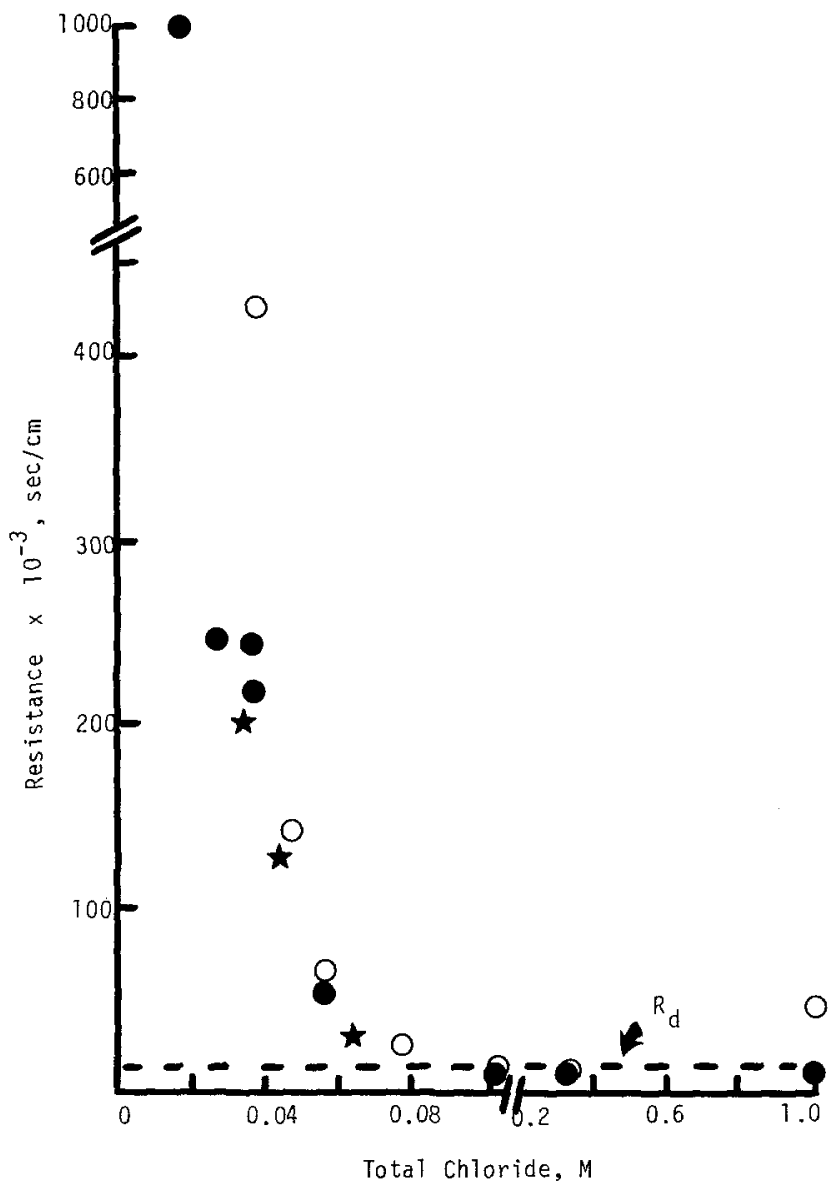

FlG. 1. Change in total mass transfer resistance with total chloride counterion concentration for cholesterol monohydrate dissolution in Renex- 690 and benzalkonium chloride solutions. Key: $1 \%$ Renex-690,6.8 mM BC; $\star 2 \%$ Renex-690, $13.6 \mathrm{~m} M$ BC; O, 4\% Renex-690, 27.2 mM BC.

kinetics data to obtain a quantitative description of the interfacial barrier in terms of electrical double layer parameters, surface potentials, dispersion force constants, and surface charge densities.

\section{DESCRIPTION OF THE PHYSICAL MODEL}

The approach of charged micelles to the charged surface of a cholesterol pellet can be considered as a phenomenon of diffusion within an electrical field across the aqueous boundary layer (Fig. 4). The steady-state flux is

$$
J_{\mathrm{m}}=\frac{D\left(M_{\mathrm{b}}-M_{\mathrm{s}}\right)}{\int_{0}^{h} \exp (V / k T) d x}=\frac{D\left(M_{\mathrm{b}}-M_{\mathrm{s}}\right)}{h W}
$$

$$
W=\frac{1}{h} \int_{0}^{h} \exp (V / k T) d x \quad(W \geqslant 1.0)
$$

where $J_{\mathrm{m}}=$ flux of free micelles (noncholesterol-loaded micelles) per unit area; $M_{\mathrm{b}}, M_{\mathrm{s}}$ $=$ micelle concentrations in the bulk solution and at the membrane surface, respectively; $D=$ diffusion coefficient of the micelle; $h=$ thickness of the aqueous boundary layer; $W=$ dimensionless constant accounting for the potential energy barrier; $k T=$ thermal energy.

The total potential energy of the system, $V$, is the sum of the repulsion and attraction energies:

$$
V=V_{\mathrm{R}}+V_{\mathrm{A}}
$$




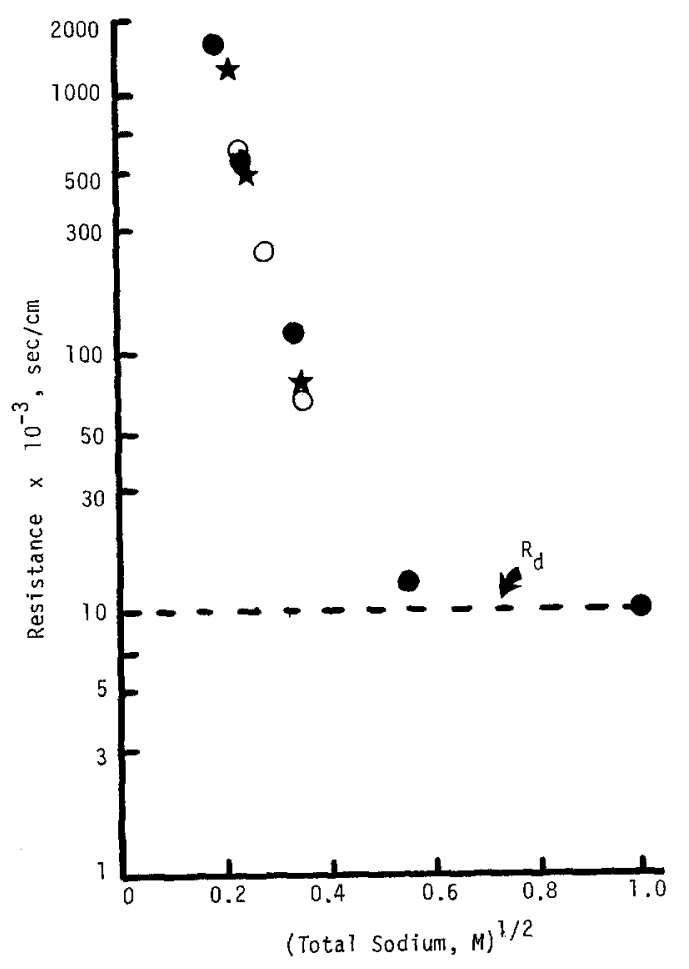

FIG. 2. Relationship between logarithm of total mass transfer resistance, $R$, with the square-root molar concentration of total sodium for cholesterol monohydrate dissolution data at several levels of total solubilizer concentration. Renex-690-to-sodium dodecyl sulfate weight ratio was kept constant at 5.0. Key: - 1\% Renex690, 6.8 mM SDS: $\star, 2 \%$ Renex-690, $13.6 \mathrm{~m} M$ SDS; O, 4\% Renex-690, $27.2 \mathrm{~m} M$ SDS.

The potential energy of repulsion, $V_{R}$, due to the overlapping of electrical double layers between a sphere and flat plate, is approximated by

$$
\begin{gathered}
V_{\mathrm{R}}=\frac{\epsilon a\left(\psi_{\mathrm{m}}+\psi_{\mathrm{s}}\right)^{2}}{4} \ln \left(1+e^{-\kappa x}\right) \\
(\kappa a>1),
\end{gathered}
$$

where $\epsilon=$ dielectric constant of the liquid medium; $a=$ radius of the micelle, $\mathrm{cm} ; \psi_{\mathrm{m}}$, $\psi_{\mathrm{s}}=$ the surface potentials of the micelle and cholesterol in statvolt, respectively; $\kappa=$ the Debye-Hückel reciprocal thickness of the electrical double layer, $\mathrm{cm}^{-1} ; x=$ the closest distance of separation between the sphere and flat surface. The potential energy of attraction, $V_{\mathrm{A}}$, due to dispersion forces is

$$
V_{\mathrm{A}}=-\frac{A a}{6 x}\left(\frac{\lambda}{\lambda+3.54 \pi x}\right)
$$

where $A=$ net Hamaker's energy constant of the system, erg, and $\lambda=$ characteristic wavelength of the atoms taken usually as $10^{-5} \mathrm{~cm}$.

The flux for the dissolution of cholesterol by micelles per unit area under sink conditions is

$$
J=\frac{C_{\mathrm{s}}}{R},
$$

where $C_{\mathrm{s}}=$ solubility of cholesterol in the micelles and $R=$ total mass transfer resistance. Since the flux of micelles to the surface is equal to the flux of micelle-solubilized cholesterol from the surface, i.e.,

$$
J=J_{\mathrm{m}}
$$

it is readily seen that the effective mass transfer resistance, $R(\mathrm{sec} / \mathrm{cm})$, for dissolution is

$$
R=\frac{h W}{D}=\frac{1}{D} \int_{0}^{h} \exp (V / k T) d x
$$

or

$$
\ln R=\ln \left(\frac{h}{D}\right)+\ln W \quad(W \geqslant 1.0)
$$

Here, $R$ is comprised of the resistances of the convection-diffusional aqueous boundary layer $(h / D)$ and also the interfacial barrier, which in this model is mechanistically interpreted to be electrical in nature. When $W$ $=1.0$,

$$
\ln R=\ln \left(\frac{h}{D}\right)
$$

and the dissolution rate is aqueous boundary layer controlled. When $W \gg 1.0$, the dissolution rate is controlled by the interfacial barrier in the extreme case.

Since $W$ is a complicated function of the potential energies of repulsion and attraction, which in turn are functions of $x$, an analytic solution is extremely difficult. However, ap- 


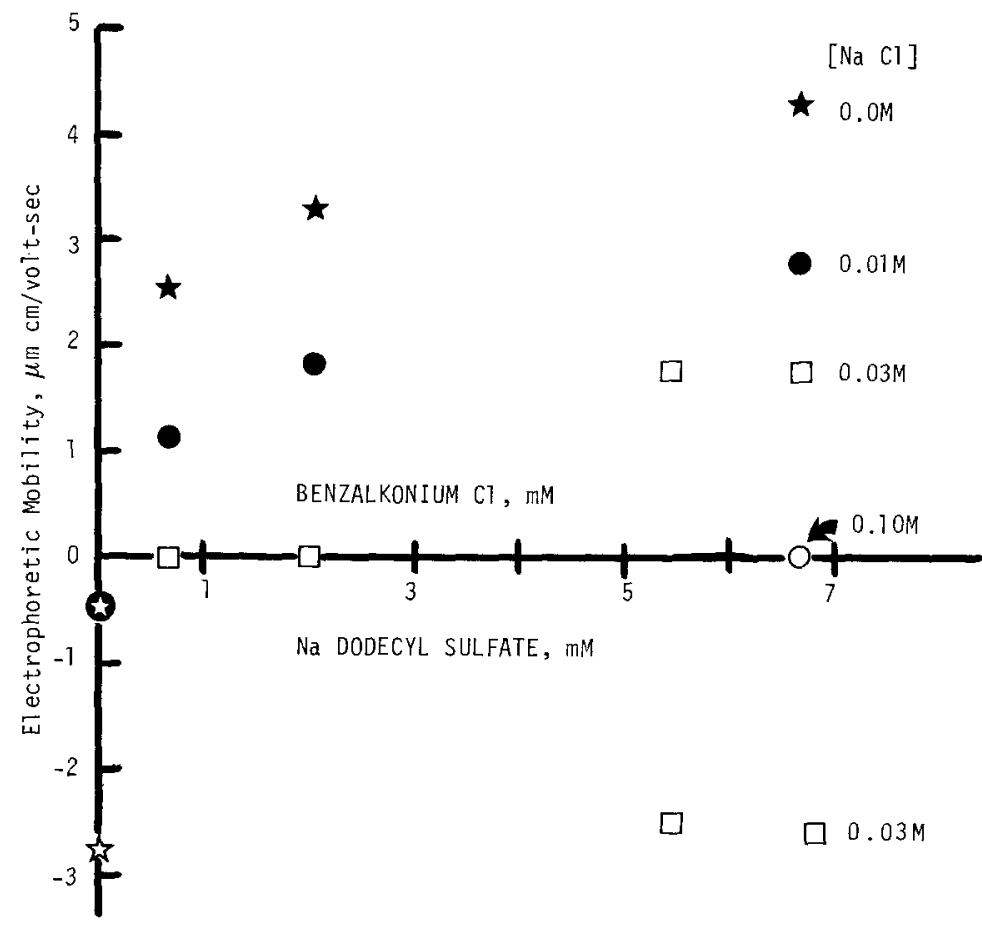

FIG. 3. The influence of ionic surfactant concentration on the electrophoretic mobility of cholesterol monohydrate crystallites in $1 \%$ Renex- 690 solutions at $25^{\circ} \mathrm{C}$ containing various concentrations of $\mathrm{NaCl}$. Key: cholesterol in water; $0,1 \%$ Renex-690 in water only.

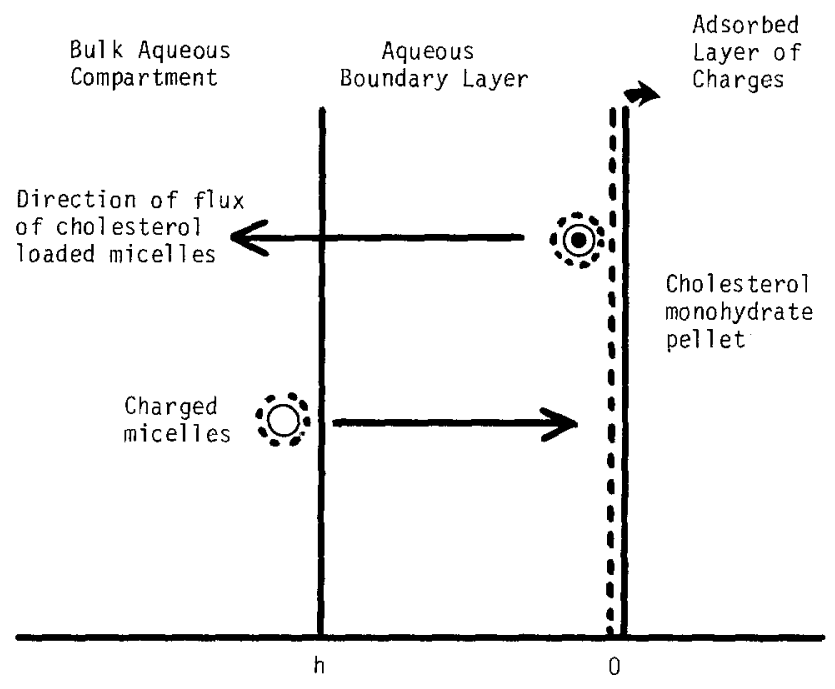

FIG. 4. Physical model for the simultaneous diffusion of charged micelles across the aqueous boundary layer within an electric field and diffusion of cholesterol monohydrate-loaded micelles away from the surface. The dotted circle around the micelle and the dotted line near the cholesterol surface are schematic depictions of the diffuse electrical double layer. 
proximate solutions are sought. This effort is desirable to enable one to quantify physically relevant parameters by graphic means. Using the Reerink and Overbeek approach, one gets

$\ln W=\frac{0.313 \beta}{k T}-\frac{1}{2} \ln \left(\frac{0.054 \beta}{k T}\right)-\frac{A a \kappa}{6 k T}$

wherein the Debye-Hückel reciprocal electrical double layer length, $\kappa\left(\mathrm{cm}^{-1}\right)$, is

$$
\kappa=\left(\frac{8 \pi e^{2} z^{2} N_{\mathrm{AV}} C}{1000 \epsilon k T}\right)^{1 / 2}
$$

for symmetrical strong electrolytes, where $e$ $=$ elementary charge, $4.805 \times 10^{-10} \mathrm{esu} ; z$ = valence; $N_{\mathrm{Av}}=$ Avogadro's number, 6.02 $\times 10^{23} ; \epsilon=$ dielectric constant of the liquid medium; $k T=$ thermal energy, erg; $C=$ electrolyte concentration in moles/liter. The $\beta$ is defined as

$$
\beta=\frac{\epsilon a\left(\psi_{\mathrm{m}}+\psi_{\mathrm{s}}\right)^{2}}{4}
$$

Finally, the substitution of Eqs. [12] and [13] into Eq. [9] yields

$$
\begin{aligned}
\ln R=\ln \left(\frac{h}{D}\right) & +\frac{0.313 \beta}{k T}-\frac{1}{2} \ln \left(\frac{0.054 \beta}{k T}\right) \\
& -\frac{A a}{6 k T}\left(\frac{8 \pi e^{2} z^{2} N_{\mathrm{AV}}}{1000 \epsilon k T}\right)^{1 / 2} \sqrt{C}
\end{aligned}
$$

A linear relationship of $\ln R$ versus $\sqrt{C}$ is predicted from which the net Hamaker's constant $A$ can be estimated from the slope and the effective surface potential term $\left(\psi_{\mathrm{m}}\right.$ $\left.+\psi_{s}\right)^{2}$, from the intercept by extrapolation.

\section{PHYSICAL MODEL TREATMENT OF THE DATA}

\section{Renex-690/Benzalkonium Chloride/ Cholesterol System}

The plot of the total mass transfer resistance, $R$, with the molar concentration of chloride counterions is shown in Fig. 1. Here, the total chloride concentration includes the contribution of chloride species from sodium chloride and benzalkonium chloride. The minimum resistance (plateau region) is 10 $\times 10^{3} \mathrm{sec} / \mathrm{cm}$. Hence, with $D=4.7 \times 10^{-7}$ $\mathrm{cm}^{2} / \mathrm{sec}$ in water at $37^{\circ} \mathrm{C}$, the effective aqueous boundary layer thickness $h$ is about $50 \mu \mathrm{m}$, which is very reasonable under the well-stirred conditions employed in the dissolution experiments. From the Stokes-Einstein equation relating the diffusion coefficient to particle size, the estimated micelle radius is $69 \AA$.

The ensuing physical model treatment will be restricted to the $1 \%$ Renex-690 and 6.8 $\mathrm{m} M$ benzalkonium chloride system since the data are more complete for analysis. Although a quantitative description of the interfacial barrier to cholesterol dissolution is summarized in Table I the method of approach will, henceforth, be discussed.

From the least-square analysis of the $\ln R$ versus $\sqrt{C}$ data, the slope and intercept are found to be 22.018 and 16.44 , respectively. It is seen from Eq. [14] that

$$
\frac{A a}{6 k T}\left(\frac{8 \pi e^{2} z^{2} N_{\mathrm{AV}}}{1000 \epsilon k T}\right)^{1 / 2}=22.02
$$

from which, with the micelle radius being 69 $\AA$ and $T$ being $310.15^{\circ} \mathrm{K}$, the net Hamaker's dispersion constant $A$ is $2.47 \times 10^{-13}$ erg. Generally, Hamaker constants range from $10^{-12} \mathrm{erg}$ for inorganic sols to $10^{-14} \mathrm{erg}$ for aqueous sols of hydrocarbon substances (2). Thus, the estimated constant for the Renex$690 /$ benzalkonium chloride/cholesterol system seems reasonable.

The net surface potential $\left(\psi_{\mathrm{m}}+\psi_{\mathrm{s}}\right)$, for the charged micelle and cholesterol system was found from the intercept:

$$
\ln \left(\frac{h}{D}\right)+\frac{0.313 \beta}{k T}-\frac{1}{2} \ln \left(\frac{0.054 \beta}{k T}\right)=16.64
$$

whereupon, by successive approximation, $\beta$ is estimated to be $1.034 \times 10^{-12} \mathrm{erg}$ and, in turn, the parameter $\left(\psi_{\mathrm{m}}+\psi_{\mathrm{s}}\right)$ is readily found to be $2.85 \times 10^{-4} \mathrm{stat} V$ or about $85.5 \mathrm{mV}$ with the aid of Eq. [13]. It has been assumed here that the diffusion coefficient of the charged micelle is not too different within the range of counterion concentrations em- 
TABLE I

Values of Various Parameters Obtained Based on the Physical Model Treatment of the Data for $1 \%$ Renex-690/6.8 $\mathrm{m} M$ Benzalkonium Chloride/Cholesterol System

\begin{tabular}{ll}
\multicolumn{1}{c}{ Description } & \multicolumn{1}{c}{ Quantity } \\
\hline 1. General parameters & \\
Graphical data & 22.02 \\
$\quad$ a. Slope & 16.64 \\
$\quad$ b. Intercept & 0.98 \\
$\quad$ c. Correlation coefficient & $2.47 \times 10^{-13} \mathrm{erg}$ \\
Hamaker's constant, $A$ & $2.85 \times 10^{-4} \mathrm{stat} \mathrm{V}(85.5 \mathrm{mV})$ \\
Net surface potentials, $\psi_{\mathrm{m}}+\psi_{\mathrm{s}}$ & \\
2. Cholesterol monohydrate surface & $4.25 \frac{\mu \mathrm{sec}^{-1}}{\mathrm{~V} / \mathrm{cm}^{-1} \pm 0.15 \mathrm{SD}}$ \\
Electrophoretic mobility of ChM $/ 6.8 \mathrm{~m} M$ & $1.83 \times 10^{-4} \mathrm{stat} \mathrm{V}(55 \pm 2.2 \mathrm{mV})$ \\
BC/1\% Renex-690/0.0 M NaCl & $3.5 \times 10^{3} \mathrm{esu} / \mathrm{cm}^{2}\left(7.3 \times 10^{12} \mathrm{charges} / \mathrm{cm}^{2}\right)$ \\
Surface potentials, $\psi_{\mathrm{s}}$ & $1.02 \times 10^{-4} \mathrm{stat} \mathrm{V}(30.5 \pm 2.2 \mathrm{mV})$ \\
Surface charge density, $\sigma_{\mathrm{s}}$ & $2.52 \times 10^{3} \mathrm{esu} / \mathrm{cm}^{2}\left(5.24 \times 10^{12} \mathrm{charges} / \mathrm{cm}^{2}\right)$ \\
3. Renex-690/BC Micelle & $32 \pm 1 \mathrm{charges}$ \\
Surface potential, $\psi_{\mathrm{m}}$ & 74 molecules \\
Surface charge density, $\sigma_{\mathrm{m}}$ & \\
Total charges/micelle & \\
Renex-690 molecules/micelle &
\end{tabular}

ployed here. Next, we will attempt to quantify $\psi_{\mathrm{s}}$ and $\psi_{\mathrm{m}}$.

To estimate the potential on the charged cholesterol surface, $\psi_{\mathrm{s}}$, due to adsorbed surfactants, the electrophoretic mobility studies of cholesterol in the presence of $1 \%$ Renex690 and $6.8 \mathrm{mM} \mathrm{BC}$ and absence of sodium chloride (Fig. 3) are used. The electrophoretic mobility is $4.25 \mu \cdot \mathrm{cm} / \mathrm{V}-\mathrm{sec} \pm 0.15 \mathrm{sd}$. As a first approximation, the Smoluchowski equation is used to calculate the zeta potential:

$$
\psi_{z}=\frac{4 \pi \eta}{\epsilon}\left(\frac{\nu}{E}\right) \quad(\kappa a \geqslant 300)
$$

where $\psi_{z}$ is the zeta potential in statvolts; $\eta$ is the viscosity of the medium $(P)$ taken as water; $\epsilon$ is the dielectric constant of the medium; $\nu$ is the velocity of the particle $(\mathrm{cm} /$ sec) in the applied electric field $E ; \nu / E$ is the electrophoretic mobility $\left(\mathrm{cm}^{2} / \mathrm{statV}\right.$-sec). Thus $\psi_{z}$ is estimated to be $1.83 \times 10^{-4}$ statV or $55 \mathrm{mV}$.

There are inherent basic assumptions in the use of Eq. [15] to calculate the zeta potential of small cholesterol crystals with adsorbed surfactants and to apply this value to the potential at the flat surface of the compressed cholesterol pellet of the dissolution experiments. The equation is said to hold for any particle shape provided the effective radius of curvature of the particle is larger by 300 times the thickness of the electrical double layer, $1 / k(3,4)$. The latter restriction takes into account that the electrophoretic retardation or drag caused by the deformation of the electrical double layer and displacement of the double layer opposite to the movement of the particle in the applied electric field, is minimal when $\kappa a \geqslant 300$. Since cholesterol crystals are flat plates (its ideal counterpart being an oblate ellipsoid of revolution with a large major to minor axial ratio) and the Debye-Hückel parameter $\kappa$ is $2.74 \times 10^{6} \mathrm{~cm}^{-1}$ in the $1 \%$ Renex-690 and $6.8 \mathrm{~m} M$ benzalkonium chloride system without supporting sodium chloride electrolyte, the condition of $k a$ about the flat surface can be assumed to be fulfilled. Edge considerations are neglected. Furthermore, considering the geometry of the cholesterol crystals, one would expect the hydrodynamic motion and frictional coefficient to be different from an 
equivalent sphere. However, Henry (3) gave theoretical justification to Smoluchowski's equation for nonelectrophoretically retarded, nonconducting, cylinders regardless of its hydrodynamic orientation and also discussed the experimental evidence in support of the theoretical conclusions (see also Ref. (4)). Owing to the quaternary ammonium structure of the surface potential-determining benzalkonium ions and also its orientation and position within the voluminous mantle of highly hydrated polyoxyethylene chains of the Renex-690 molecules adsorbed on the cholesterol surface, the charge would be somewhat shielded from bulk water (1). Thus, the zeta potential of $55 \mathrm{mV}$ in the absence of sodium chloride is a measure of surface potential of the Stern-type layer. Since this Stern-type layer is likely to be the same on the compressed cholesterol pellet, it seems appropriate that the potential measured for the Renex-690/BC/cholesterol crystal system could be used to approximate the electrical potential in the cholesterol dissolution experiments.

The surface charge density of the flat cholesterol plate is estimated by

$$
\sigma_{\mathrm{s}}=\left(\frac{\epsilon k T N_{\mathrm{AV}} C}{500 \pi}\right)^{1 / 2} \sinh \left(\frac{z e \psi_{\mathrm{s}}}{2 k T}\right),
$$

where $\sigma_{\mathrm{s}}$ is the surface charge density in statCoulombs per square centimeter and the other terms have already been defined. Furthermore,

$$
\text { No. of charges per } \mathrm{cm}^{2}=\frac{\sigma_{\mathrm{s}}}{e},
$$

where $e$ is elementary charge, $4.805 \times 10^{-10}$ esu, and the

$$
\text { Total number of charges }=\frac{\pi r^{2} \sigma_{s}}{e},
$$

where $\pi r^{2}$ is the surface area of the cholesterol disk $\left(1.267 \mathrm{~cm}^{2}\right)$ employed in the dissolution studies. Using Eqs. [16] and [17], the surface charge density is $7.29 \times 10^{12}$ charges $/ \mathrm{cm}^{2}$. The charges originate from adsorbed benzalkonium cations.

Journal of Colloid and Interface Science, Vol, 101, No. 2, October 1984
Since $\left(\psi_{\mathrm{s}}+\psi_{\mathrm{m}}\right)$ was found to be $85.5 \mathrm{mV}$ and $\psi_{\mathrm{s}}$ to be $55 \mathrm{mV}$, then the surface potential of the Renex-690/BC micelle is $30.5 \mathrm{mV}$ by difference. It is noted that, with $k$ being 2.74 $\times 10^{6} \mathrm{~cm}^{-1}$ and the micelle radius $a$ being $69 \AA$, the product $\kappa a$ is larger than unity. In pursuing calculations on the surface charge density of the micelle, an approximate expression can be used:

$$
\begin{aligned}
\sigma_{\mathrm{m}} & =\frac{\epsilon \kappa \psi_{\mathrm{m}}}{4 \pi}\left(1+\frac{1}{\kappa a}\right) \\
& =5.24 \times 10^{12} \text { charges } / \mathrm{cm}^{2} .
\end{aligned}
$$

Consequently, the total benzalkonium ions per micelle are 32 charges. When one employs the rigorous numerical approach of Loeb et al. (5), $\sigma_{\mathrm{m}}$ is equal to $5.54 \times 10^{12}$ charges/ $\mathrm{cm}^{2}$ and the total charges per micelle are 33 .

To gain further insights into the molecular composition of the mixed micelle, calculations were made to estimate the number of Renex-690 molecules per micelle (Appendix B). After delineating the total number of benzalkonium ions in the system into the number of ions associated with the cholesterol surface and micelle, the total number of micelles were obtained; whereupon, subsequent calculations accounting for the nonionic surfactant indicate that there are 74 Renex-690 and 31 benzalkonium molecules per micelle. Interestingly, pure Renex- 690 micelles have an aggregation number of $100(6)$.

\section{Renex-690/Na Dodecyl Sulfate/ Cholesterol System}

Employing the identical approach to the data analysis as in the previous section, various phenomenological parameters of the physical model were calculated. These are summarized in Table II. The effective Hamaker's attraction constant is $2.19 \times 10^{-13}$ erg, and the net potential $\left(\psi_{\mathrm{m}}+\psi_{\mathrm{s}}\right)$, at infinite sodium chloride dilution is $92.7 \mathrm{mV}$.

The use of the zeta potential of cholesterol crystals with adsorbed potential-determining dodecyl sulfate ions together with Renex-690 
TABLE II

Values of Various Parameters Obtained based on the Physical Model Treatment of the Data for Renex-690/Sodium Dodecyl Sulfate/Cholesterol System

\begin{tabular}{ll}
\hline \multicolumn{1}{c}{ Description } & \multicolumn{1}{c}{ Quantity } \\
\hline 1. General parameters & \\
Graphical data & 19.57 \\
a. Slope & 18.02 \\
$\quad$ b. Intercept & 0.996 \\
$\quad$ c. Correlation coefficient & $2.19 \times 10^{-13} \mathrm{erg}$ \\
Hamaker's constant, $A$ & $3.09 \times 10^{-4} \mathrm{stat} \mathrm{V}(92.7 \mathrm{mV})$ \\
Net surface potentials, $\psi_{\mathrm{m}}+\psi_{\mathrm{s}}$ & \\
2. Cholesterol monohydrate surface & $2.19 \times 10^{-4} \mathrm{stat} \mathrm{V}(65.6 \pm 3.2 \mathrm{mV})$ \\
Surface potentials, $\psi_{\mathrm{s}}$ at $0.0 \mathrm{M} \mathrm{NaCl}$ & $4.49 \times 10^{3} \mathrm{esu} / \mathrm{cm}^{2}$ \\
Surface charge density, $\sigma_{\mathrm{s}}$ & $9.34 \times 10^{12} \mathrm{charges} / \mathrm{cm}^{2}$ \\
& \\
3. Renex-690/SDS micelle & $9.02 \times 10^{-5} \mathrm{stat} \mathrm{V}(27.1 \pm 3.2 \mathrm{mV})$ \\
Surface potential, $\psi_{\mathrm{m}}$ & $2.23 \times 10^{3} \mathrm{esu} / \mathrm{cm}^{2}\left(4.63 \times 10^{12} \mathrm{charges} / \mathrm{cm}^{2}\right)$ \\
Surface charge density, $\sigma_{\mathrm{m}}$ & $29 \pm 1 \mathrm{charges}$ \\
Total charges $/$ micelle & $65 \mathrm{molecules}$ \\
Renex-690 molecules $/$ micelle & \\
\hline
\end{tabular}

surfactant to estimate $\psi_{\mathrm{s}}$ on the cholesterol pellet was complicated by the availability of electrophoretic measurements of cholesterol crystals only in $1 \%$ Renex-690, 6.8 $\mathrm{mM}$ sodium dodecyl sulfate and $0.03 \mathrm{M} \mathrm{NaCl}$ solution. For this system, the electrophoretic mobility and $\psi_{z}$ were $2.6 \mu \mathrm{cm} / \mathrm{V}$-sec and $33.4 \mathrm{mV}$ at $25^{\circ} \mathrm{C}$, respectively. Using Eqs. [16] and [17], the surface charge density $\sigma_{\mathrm{s}}$ is found to be $4.49 \times 10^{3} \mathrm{esu} / \mathrm{cm}^{2}$ or 9.34 $\times 10^{12}$ charges $/ \mathrm{cm}^{2}$. Next, to calculate $\psi_{\mathrm{s}}$ at infinite sodium chloride dilution, the $\sigma_{\mathrm{s}}$ estimated from the zeta potential at $0.03 \mathrm{M}$ $\mathrm{NaCl}$ was assumed to be constant for all sodium chloride concentrations. Equation [16] was then rearranged to yield $\psi_{\mathrm{s}}$ as

$$
\psi_{\mathrm{s}}=\frac{2 k T}{z e} \sinh ^{-1}\left[\sigma_{\mathrm{s}}\left(\frac{500 \pi}{\epsilon k T N_{\mathrm{av}} C}\right)^{1 / 2}\right] \text {, }
$$

where $C$ is equal to $6.8 \times 10^{-3} M$ sodium ions. Consequently, $\psi_{\mathrm{s}}$ is $2.19 \times 10^{-4} \mathrm{statV}$ or $65.6 \mathrm{mV}$.

The potential on the mixed micelle $\psi_{\mathrm{m}}$ is estimated to be $27.1 \mathrm{mV}$ whereupon the surface charge density $\sigma_{\mathrm{s}}$ is $4.83 \times 10^{12}$ charges $/ \mathrm{cm}^{2}$ or 29 charges per micelle. Fur- thermore, there are about 65 molecules of Renex-690 in the mixed micelle.

\section{APPENDIX A}

\section{A. Linear Approximation of the W Factor}

The transport resistance describing the diffusion of a charged sphere to a charged flat plate was described in part by the dimensionless $W$ factor in Eq. [2], i.e.,

$$
W=\frac{1}{h} \int_{0}^{h} \exp (V / k t) d x \geqslant 1.0
$$

The definitions of the terms here and henceforth are found in the main text. The potential energy of interaction between the sphere and plate is given by the sum of the repulsive energy due to the overlap of electrical double layers and the dispersion energy of attraction:

$$
V=\frac{\epsilon a\left(\psi_{\mathrm{s}}+\psi_{\mathrm{m}}\right)^{2}}{4} \ln \left(1+e^{-\kappa x}\right)-\frac{A a}{6 x} .
$$

The first term on the right side of the equation is an approximation of the more rigorous and complicated expression for the repulsive energy between a sphere and plate of dissim- 
ilar surface potentials (7). The retardation effect on the attraction energy is neglected since the effect will be inconsequential when the energy is evaluated at $x=1 / \kappa$, the electrical double layer thickness.

We seek a linear approximation to [A-1] utilizing the Reerink and Overbeek approach (8). Since $W$ is mainly determined by the maximum of the Gaussian-like curve of (1/ $h) \cdot \exp (V / k T)$ versus $x$ where $V=V_{\max }$ at $x \simeq 1 / \kappa$, the electrical double layer thickness, the potential function $V$ could be expanded at the primary barrier $V_{\max }$ by Taylor series:

$$
\begin{aligned}
V=V_{\max }+(\Delta x) & V_{\max }^{\prime} \\
& +\frac{(\Delta x)^{2}}{2} V_{\max }^{\prime \prime}+\cdots
\end{aligned}
$$

where $(\Delta x) V_{\max }^{\prime}=0$ since the derivative $V_{\max }^{\prime}=0$, and the higher order terms are negligibly small. Upon substituting [A-3] into [A-1] and replacing the upper and lower limits of integration by $+\infty$ and $-\infty$, respectively, the original curve is replaced by a Gauss curve with the same height and curvature about the maximum. Thus,

$$
\begin{aligned}
W=\frac{e^{V_{\max } / k T}}{h} \int_{-\infty}^{\infty} & \\
& \times \exp \left[-p^{2}(\Delta x)^{2}\right] d(\Delta x)
\end{aligned}
$$

and

$$
p^{2}=-V_{\max }^{\prime \prime} / 2 k T
$$

Equation [A-4] is a definite integral in standard form whereupon the solution is

$$
W=\frac{e^{V_{\max } / k T}}{h} \frac{\sqrt{\pi}}{p}
$$

and

$$
\ln W=\ln \frac{\sqrt{\pi}}{h}-\ln p+\frac{V_{\max }}{k T} .
$$

We now strive to obtain an expression for $p$ as described by Eq. [A-5]. With the use of Eq. [A-2], it follows that

$$
\left.V^{\prime}\right|_{x=1 / \kappa}=V_{\max }^{\prime}=0
$$

and, subsequently,

$$
0.27 \beta=\frac{A a_{\kappa}}{6},
$$

where

$$
\beta=\frac{\epsilon a\left(\psi_{\mathrm{s}}+\psi_{\mathrm{m}}\right)^{2}}{4}
$$

It also follows that

$$
V_{\max }^{\prime \prime}=0.2 \beta \kappa^{2}-\frac{A a \kappa^{3}}{3}
$$

and with Eq. [A-9], we get

whereupon

$$
V_{\max }^{\prime \prime}=-0.34 \beta \kappa^{2}
$$

$$
p=\left(\frac{0.17 \beta \kappa^{2}}{k T}\right)^{1 / 2}
$$

Finally, the substitutions of Eq. [A-13] and the following expressions,

$$
\begin{aligned}
V_{\max } & =0.313 \beta-\frac{A a \kappa}{6} \\
\kappa & =\left(\frac{8 \pi e^{2} z^{2} N_{\mathrm{AV} C}}{1000 \epsilon k T}\right)^{1 / 2}
\end{aligned}
$$

into Eq. $[\mathrm{A}-7]$ yields

$$
\begin{aligned}
\ln W= & \frac{0.313 \beta}{k T}-\frac{1}{2} \ln \left(\frac{0.054 \beta}{k T}\right) \\
& -\frac{A a}{6 k T}\left(\frac{8 \pi e^{2} z^{2} N_{\mathrm{AV}}}{1000 \epsilon k T}\right)^{1 / 2} \sqrt{C}
\end{aligned}
$$

We have assumed here that $\kappa h \simeq 1$ when $\sqrt{C}$ approaches zero.

\section{APPENDIX B}

\section{B. Number of Renex-690 Molecules per Micelle}

To estimate the number of Renex-690 molecules per micelle of mixed composition in $1 \%$ Renex-690/6.8 $\mathrm{m} M$ ionic surfactant solutions, we first calculate the total number of micelles in the system from mass balance considerations using known quantities for the ionic surfactants. 


$$
N_{\text {micelles }}=\frac{Q_{\text {total }}-Q_{\mathrm{s}} A}{Q_{\mathrm{m}}},
$$

where $N_{\text {micelles }}=$ total number of micelles; $Q_{\text {total }}=$ total number of ionic surfactant molecules, i.e., the product of surfactant concentration $(6.8 \mathrm{~m} M)$ and the solution volume $(2 \mathrm{ml}) ; Q_{\mathrm{s}}=$ number of ionic surfactant molecules adsorbed on the cholesterol surface per unit area; $Q_{\mathrm{m}}=$ number of ionic surfactant molecules per micelle, i.e., the product of surface charge density (see Eq. [19]) and the micelle surface area; $A=$ area of exposed pellet surface which is $1.27 \mathrm{~cm}^{2}$. For the 6.8 $\mathrm{m} M$ benzalkonium chloride or sodium dodecyl sulfate solution containing $1 \%$ Renex690 , we estimate about $2.48 \times 10^{17}$ and 2.82 $\times 10^{17}$ micelles, respectively, in the $2-\mathrm{ml}$ solution.

With respect to Renex-690 micelle composition,

$$
R_{\mathrm{m}}=R_{\text {total }} / N_{\text {micelles }}
$$

where $R_{\mathrm{m}}=$ mass of Renex-690 per micelle; and $R_{\text {total }}=$ total mass in the system, i.e., the product of the concentration ( $1 \% \mathrm{w} / \mathrm{v}$ Renex$690)$ and the solution volume $(2 \mathrm{ml})$. Therefore,
Number of Renex-690 molecules per micelle

$$
=\frac{R_{\mathrm{m}} N_{\mathrm{AV}}}{(M W)},
$$

where $M W=$ average molecular weight which is 660 ; and $N_{\mathrm{AV}}=$ Avogadro's number. Here, we have assumed that the mass of Renex690 adsorbed to the cholesterol surface is negligible.

\section{REFERENCES}

1. Gupta, S. L., Higuchi, W. I., and Ho, N. F. H., J. Colloid Interface Sci., in press.

2. Fowkes, F. M., "Chemistry and Physics of Interfaces." Amer. Chem. Soc., Washington, D.C., 1965.

3. Henry, D. C., Proc. Roy. Soc. Ser. A 133, 106 (1931).

4. Overbeek, T. T. H. G., in "Colloid Science," (H. R. Kruyt, Ed.), p. 115. Elsevier, The Netherlands, 1952.

5. Loeb, A. L., Overbeek, J. T. H. G., and Wiersema, P. H., in "The Electrical Double Layer Around a Spherical Colloid Particle." The MIT Press, Mass., 1961.

6. Becher, P., J. Colloid Sci. 16, 49 (1961).

7. Hogg, R., Healy, T. W., and Fuerstenau, D. W., Trans. Faraday Soc. 62, 1638 (1966).

8. Reerink, H., and Overbeek, J. T. H. G., Discuss. Faraday Soc. 18, 74 (1954). 